\title{
VALORACIÓN DE LAS ACTUACIONES DE ATENCIÓN EDUCATIVA AL ALUMNADO INMIGRANTE EN CASTILLA Y LEÓN
}

\author{
Rufino Cano González \\ Universidad de Valladolid
}

\begin{abstract}
RESUMEN: Las comunidades sociales de nuestro entorno, y la nuestra no es una excepción, se distinguen por la convivencia en su seno de una progresiva y creciente diversidad de culturas y valores. El fenómeno de la inmigración no es algo que exista y permanezca al margen de nuestras fronteras. La integración social y educativa del inmigrante se ha convertido para el Gobierno de la Comunidad de Castilla y León en una cuestión de interés general y de atención preferente desde todos los ámbitos de la Administración autonómica. La escuela, en tanto que una de las instancias con más responsabilidad de la sociedad, no es ajena a la influencia de los distintos factores sociales y culturales sino que, muy al contrario, se muestra muy sensible al fenómeno de la multiculturalidad. A analizar y valorar sus experiencias y prácticas de educación intercultural, hemos dedicado este artículo.
\end{abstract}

ABSTRACT: The social communities surrounding us are characterized by a progressive growth of diverse cultures and values living together at their centre. In this aspect, our own community is no exception. The phenomenon of immigration is not something which exists only outside our borders. The social integration and education of immigrants has become a question of social interest which demands preferential attention from all administrative areas of the Government of Castilla y León. School, as one of the most responsible instances of society, is directly influenced by social and cultural factors and is also sensitive to the phenomenon of a multicultural society. We have devoted this article to analyzing and evaluating experiences and practices of intercultural education in schools.

\section{INTRODUCCIÓN}

La inmigración, desde el cumplimiento de la norma democrática legalmente establecida, es un derecho de todo ciudadano; fuera de esta dimensión política, la inmigración no regularizada, en el mejor de los supuestos posibles, es un derecho 
moral, muchas veces adulterado por distintos motivos interesados y hasta punibles, lo que, en absoluto, resta un ápice a nuestra posición favorable a este hecho.

La sociedad castellano-leonesa ha experimentado, durante los últimos años, un viraje en su configuración (que no está siendo impulsivo ni drástico, en la medida en que la población inmigrante asentada no representa un porcentaje tan elevado como en otras Comunidades Autónomas, aunque sí se muestra significativo), un cambio importante en cuanto a su composición debido, entre otras circunstancias, al aumento de la inmigración que los flujos migratorios han venido incrementando movidos por distintas razones generalmente muy entendibles desde perspectivas diferentes. El actual concepto de sociedad, planteado y entendido como sistema de relaciones a través del cual unos individuos se conectan con otros, y todos entre sí, está cambiando ya que, actualmente, en nuestra sociedad converge una pluralidad de personas de una variada procedencia de culturas, creencias, etc. La integración educativa y social del inmigrante se ha convertido, pues, en una realidad trascendental, en una cuestión de interés general y abordarla es un tema objeto de debate y consenso.

El presente artículo responde al interés por conocer y valorar, de manera global, el conjunto de las actuaciones y experiencias de "atención educativa al alumnado inmigrante" Ilevadas a cabo, en contextos integradores, a lo largo y ancho de la compleja y variada geografía multifacial de la Comunidad de Castilla y León, tanto desde el punto de vista de la literatura generada por la Administración como desde la producción de prácticas escolares que se vienen gestando e implementando, como ejemplo, en algunos de sus centros de Educación Primaria y Secundaria especialmente comprometidos con esta enriquecedora tarea docente de atención a la diversidad. El fin, a muy corto plazo, no es otro que ir elaborando ya, sin demora, respuestas pedagógico-didáctico-organizativas mejor planificadas, más flexibles, completas y dinámicas desde el punto de vista del currículo, -atendiendo a la complejísima variedad que aparece en nuestras aulas y centros-, en mayor conexión con las recomendaciones e idoneidad de experiencias realizadas y resultados obtenidos, profundamente enraizadas e integradas en el conjunto total de las prácticas escolares desde una perspectiva marcadamente normalizadora, transversalizadora y, a la vez, comprensiva para todos los alumnos, -autóctonos e inmigrantes-, en todas las acciones ordinarias y extraordinarias del horario escolar con independencia de su lengua materna, su cultura, su condición social y origen.

\section{Aspectos Cualitativos y CUANtitativos de la fotografía migratoria en CAstilla y LeÓN}

La panorámica actual de la fotografía migratoria que afecta a la Europa Occidental presenta, ciertamente, connotaciones y características particulares relacionadas directamente con: a) las proporciones reales y magnitudes irreversibles que este proceso, en continuo crecimiento en cadena, está adquiriendo en el espacio desde contextos geográficos amplios (naciones, ciudades importantes, capitales de provincia) hasta espacios territoriales de pequeñas dimensiones demográficas (pequeñas pobla- 
ciones, pueblos, aldeas); b) la rapidez vertiginosa con la que este acaecimiento se ha originado y se está desarrollando, en el tiempo, desde hace unos pocos años a estas fechas; c) el ritmo tan exageradamente acelerado y desbordante en el que se está produciendo este crecimiento de tan preocupantes magnitudes de todo tipo; d) la gran variedad y diversidad de los países de origen de los inmigrantes que se insertan en nuestras sociedades; e) la complicada red de culturas que se dan cita y confluyen, con efecto multiplicador, en espacios de creciente diversidad cultural y, queremos suponer, de convivencia democrática fundamentada en derechos y deberes universales; f) la aparición de amplios contextos y grupos diferenciales de carácter marcadamente multicultural como consecuencia de "asentamientos subterráneos", ciertamente, ocultos y poco organizados desde parámetros sociales; g) el rompimiento de los límites de las fronteras y, por tanto, a la eliminación de las cercas culturales circunscritas, hasta ahora, a sus ámbitos de influencia predominante, creando, de esta manera, al menos teóricamente, situaciones potencialmente enriquecedoras y de una mayor complejidad.

Castilla y León, en el marco de las competencias que recoge el Estatuto de Autonomía, y de acuerdo con cuantas normas y principios internacionales, estatales y comunitarios, abordan este tema, viene desarrollando, con la implicación de distintas Consejerías (Consejería de Presidencia y Administración Territorial, de Economía y Empleo, de Familia e Igualdad de Oportunidades, de Educación, de Sanidad, de Fomento, de Cultura y Turismo), las políticas públicas acordes con el ejercicio de los derechos y deberes de los inmigrantes. Refiriéndonos en exclusiva a la Consejería de Educación, por necesidades del tema que nos ocupa, hemos de manifestar su decidido compromiso cuando afirma que uno de los derechos de los extranjeros es el acceso en régimen de igualdad a la educación, tanto de menores como de adultos, garantizando este libre acceso y un trato no discriminatorio en los Centros educativos. Este enfoque actual de la integración educativa y social en el ejercicio de estas competencias garantiza el acceso a nuestra cultura y, a la vez, el respeto y conservación de las culturas de origen.

En consecuencia con estas afirmaciones, la importancia creciente y relevante que están adquiriendo y alcanzando estos contextos actualmente, nos obliga a afirmar, sin ningún género de dudas, que el hecho multicultural es uno de los indicadores sociales de la Europa Occidental y, más concretamente de las Comunidades integrantes del Estado Español, entre las que se encuentra Castilla y León y sus pueblos, que mayor atención demanda del conjunto de las instituciones públicas y, en particular, de las educativas, como es el caso. En este sentido, la diversidad cultural, propia de la especie humana, se está convirtiendo, en nuestros días, en una nueva dimensión social de primera magnitud, en una característica de primera importancia anexa, en general, a las sociedades occidentales, en uno de los principales factores que influyen en el comportamiento y en el proceso educativo del alumnado autóctono (cultura mayoritaria) e inmigrante (cultura minoritaria) y, por supuesto, en una especie de eje de consideración e interés fundamental dentro del conjunto de los aspectos diferenciales de la atención a la diversidad cultural y étnica. 
La integración la hemos de abordar entre todos, no como un problema o una pesadilla onerosa, imposible de superar sino, más bien, como una oportunidad de enriquecimiento cultural, social y económico, tanto para la sociedad de acogida como para los individuos que han decidido formar parte del territorio identificándose y conviviendo con sus gentes desde una doble dimensión: a) subjetiva y personal, que requiere una decisión voluntaria de integración en la sociedad receptora $y, b)$ social, que exige, por una parte, una integración efectiva y su participación en la vida comunitaria y, por otra, una la inserción en su cultura y en sus proyectos.

A continuación, ofrecemos algunos datos estadísticos del alumnado extranjero matriculado en enseñanzas no universitarias para completar y entender mejor la fotografía inmigratoria que ofrece Castilla y León desde una mirada a sus distintas provincias. Para ello, tendremos en cuenta la evolución de la población inmigrante entre los cursos 1993-94 y 2003-04, y la población que conforma los grupos de inmigración, destacando que aquella que se encuentra matriculada en este apartado lo es, fundamentalmente, proveniente de América del Sur, seguida de población aborigen de países no comunitarios y africanos. La diferencia es bastante amplia con relación a los nativos de América Central o Asia. Este hecho se analiza sobre un total de población en este sector de 9.693 alumnos/as.

A poco que nos detengamos en el visionado de los datos expuestos en esta tabla, inmediatamente observamos un fuerte incremento de alumnos de otros países repartidos por las distintas etapas educativas de nuestro sistema escolar con especial relevancia en Educación Infantil. Efectivamente, desde el curso 1993-94 se aprecia un crecimiento constantemente acelerado, con dos etapas que nos Ilaman poderosamente la atención: a) de 1993/94 a 1998-99, en la que se experimenta un crecimiento medio anual entre un $6 \%$ y un $15 \%$; b) de $1999-2000$ a 2003-04, de un rápido y vertiginoso incremento amparado en unos porcentajes en torno al $28 \%$ anual con algunos picos que superan el $45 \%$. En consecuencia, las cifras expresan con claridad que hemos pasado de 50.076 alumnos extranjeros matriculados en España en enseñanzas no universitarias en el curso 1993-94, a la nada despreciable magnitud de un total de 389.726 alumnos matriculados en el curso 2003-04. Si complementamos estos datos con las previsiones que el MEC baraja y con las conclusiones que han avanzado distintos estudios prospectivos, como es el caso de la Fundación Encuentro, hemos de afirmar que, actualmente, es posible que esta población esté muy próxima al medio millón de escolares extranjeros, cifra que corresponde a un 5,7\% del alumnado total. De ellos, el 20,3\% está matriculado en Educación Infantil, el 44,5\% (más del doble) frecuenta las aulas de Educación Primaria y un 28\% recibe en nuestras aulas Educación Secundaria no obligatoria. (Ver Tabla $n^{-1} 1$ ).

En cuanto a su procedencia, el colectivo actualmente más numeroso es el que nos Ilega de países de América del Sur y Central, sobre todo de Ecuador, Colombia y Argentina. Le siguen los inmigrantes europeos, que han superado a los africanos, con un 25,2\% (12,38\% perteneciente a países de la U.E. y 12,82\% de los países de la Europa del Este, sobre todo de Rumania, Bulgaria y Rusia) y, finalmente, los africanos que suponen un 18,9\%. (Ver Tabla nº 2). 
Tabla no 1. Evolución del alumnado extranjero matriculado en España en enseñanzas no universitarias

\begin{tabular}{|l|c|c|c|c|}
\hline \multicolumn{5}{|c|}{ CURSOS } \\
\hline \multicolumn{1}{|c|}{ TOTAL } & $1993-94$ & $1998-99$ & $2002-03$ & $2003-04$ \\
\hline E. INFANTIL/PREESCOLAR & $\mathbf{5 0 . 0 7 6}$ & $\mathbf{8 0 . 6 8 7}$ & $\mathbf{3 0 2 . 4 2 3}$ & $\mathbf{3 8 9 . 7 2 6}$ \\
\hline E. PRIMARIA/E.G.B. & 8.640 & 12.387 & 60.050 & 78.349 \\
\hline E. ESPECIAL & 32.471 & 34.017 & 132.467 & 172.888 \\
\hline E. SECUNDARIA OBLIGATORIA & - & 22.558 & 80.300 & 108.298 \\
\hline BACHILLERATO & 6.149 & 6.311 & 12.085 & 15.388 \\
\hline FORMACIÓN PROFESIONAL & 2.663 & 2.732 & 10.469 & 13.367 \\
\hline NO CONSTA ENSEÑANZA & - & 2.504 & 6.087 & - \\
\hline
\end{tabular}

FUENTE: Ministerio de Educación y Ciencia. Datos y Cifras. Curso Escolar 2004-05.

Tabla no 2. Distribución porcentual del alumnado extranjero por área geográfica de nacionalidad

\begin{tabular}{|l|c|}
\hline \multicolumn{2}{|c|}{ CURSO 2003-2004 } \\
\hline ÁREAS GEOGRÁFICAS & DISTRIBUCIÓN PORCENTUAL \\
\hline AMÉRICA DEL SUR Y CENTRAL & 50,5 \\
\hline EUROPA & 25,2 \\
\hline ÁFRICA & 18,9 \\
\hline ASIA Y OCEANÍA & 4,4 \\
\hline AMÉRICA DEL NORTE & 1,1 \\
\hline
\end{tabular}

FUENTE: Ministerio de Educación y Ciencia. Datos y Cifras. Curso Escolar 2004-05.

Si nos fijamos en las Comunidades Autónomas preferidas por los inmigrantes, hemos de concluir que el alumnado extranjero escolarizado se concentra, fundamentalmente, en las de Madrid, Illes Balears, La Rioja, Navarra, Murcia y Valencia, por citar las más pobladas. Castilla y León, aparece en un discreto 15 lugar, con un $3 \%$ repartido por sus territorios provinciales como se puede comprobar en la Tabla no 3 . 
Tabla no 3. Porcentaje de alumnado extranjero matriculado en cada Comunidad Autónoma

\begin{tabular}{|l|c|}
\hline \multicolumn{2}{|c|}{ CURSO 2003-04 } \\
\hline COMUNIDADES AUTÓNOMAS & PORCENTAJE \\
\hline MADRID & 10,2 \\
\hline ILLES BALEARS & 10,1 \\
\hline LA RIOJA & 8,1 \\
\hline NAVARRA & 7,9 \\
\hline MURCIA & 7,6 \\
\hline C. VALENCIANA & 7,5 \\
\hline CATALUÑA & 7,4 \\
\hline MELILLA & 6,5 \\
\hline CANARIAS & 6,2 \\
\hline ARAGÓN & 6,2 \\
\hline ESPAÑA & $\mathbf{5 , 7}$ \\
\hline CASTILLA-LA MANCHA & 4,2 \\
\hline CANTABRIA & 3,2 \\
\hline ANDALUCÍA & 3,1 \\
\hline CASTILLA Y LEÓN & $\mathbf{3 , 0}$ \\
\hline PAÍS VASCO & 2,5 \\
\hline ASTURIAS & 2,4 \\
\hline GALICIA & 1,7 \\
\hline EXTREMADURA & 1,6 \\
\hline CEUTA & 1,2 \\
\hline
\end{tabular}

FUENTE: Ministerio de Educación y Ciencia. Datos y Cifras. Curso Escolar 2004-05.

En Castilla y León se repite, uniformemente, la misma situación que se produce a nivel estatal respecto de la nacionalidad de procedencia del alumnado foráneo. Sigue siendo la inmigración americana, especialmente la procedente del Sur, la que ocupa el primer lugar, seguida de Europa del Este y África (Tabla ํㅡㄴ 4). 
Tabla no 4. Alumnado extranjero por continente matriculado en enseñanzas no universitarias en la Comunidad de Castilla y León

\begin{tabular}{|l|c|c|c|c|c|c|c|c|c|c|}
\hline \multicolumn{10}{|c|}{} & \multicolumn{2}{c|}{ EUROPA } & \multicolumn{3}{c|}{ AMERICA } & & & \\
\hline & TOTAL & U.E & RESTO & AFRICA & NORT & CENT & SUR & ASIA & OCEANÍA & N. CONSTA \\
\hline CASTILLA Y LEÓN & 9.693 & 1.150 & 2.188 & 1.273 & 216 & 644 & 3.802 & 372 & 4 & 44 \\
\hline ÁVILA & 535 & 14 & 79 & 76 & 6 & 41 & 306 & 8 & 2 & 3 \\
\hline BURGOS & 1.698 & 198 & 349 & 152 & 14 & 123 & 828 & 28 & - & 6 \\
\hline LEÓN & 1.555 & 359 & 185 & 185 & 21 & 82 & 604 & 115 & - & 4 \\
\hline PALENCIA & 332 & 21 & 65 & 70 & 4 & 16 & 130 & 26 & - & - \\
\hline SALAMANCA & 1.537 & 288 & 144 & 310 & 113 & 57 & 536 & 85 & 1 & 3 \\
\hline SEGOVIA & 1.387 & 31 & 647 & 222 & 4 & 102 & 353 & 23 & - & 5 \\
\hline SORIA & 648 & 21 & 94 & 88 & 5 & 73 & 333 & 28 & - & 6 \\
\hline VALLADOLID & 1.611 & 143 & 549 & 118 & 45 & 105 & 602 & 49 & - & - \\
\hline ZAMORA & 390 & 75 & 76 & 52 & 4 & 45 & 110 & 10 & 1 & 17 \\
\hline
\end{tabular}

FUENTE: Plan integral de inmigración en Castilla y León 2005-09. Consejería de Presidencia y Administración Territorial. Junta de Castilla y León, 2005, pp. 44-45.

Lo mismo ocurre cuando comparamos los porcentajes de alumnos extranjeros matriculados en centros no universitarios, a nivel del territorio nacional, en función del tipo de enseñanza, con la presencia de estos en la Comunidad de Castilla y León (Tabla no 5). Efectivamente, mientras que, a nivel nacional, el 44,5\% está matriculado en Educación Primaria, el 28\% en Educación Secundaria no obligatoria y el 20,2\% en Educación Infantil, en la Comunidad Castellano-Leonesa, estos porcentajes se sitúan, respectivamente, en el $43,2 \%$, el $26,2 \%$ y el $15,8 \%$. Por tanto, existe un cierto mimetismo entre lo que sucede a nivel de Estado y lo que está pasando en zonas territoriales más pequeñas, como es el caso.

Respecto de la distribución de los alumnos, es opinión generalizada, entre los maestros y profesores, que el alumnado autóctono e inmigrante que acude a sus Centros suele tener un nivel sociocultural similar. No obstante, en los casos en que éste es más bien bajo y, además, aparecen dificultades añadidas (conviven en familias desestructuradas, con problemas de empleo, en viviendas en malas condiciones de habitabilidad, muestran desinterés por los estudios de sus hijos) y las que provienen de ser inmigrante, entonces el Centro escolar comienza a tener dificultades y problemas reales para desarrollar su acción educativa por cuanto que las intervenciones psicopedagógicas suelen ser muy específicas e individualizadas.

Otra variable a tener en cuenta viene determinada por los espacios de concentración territorial en los que fijan su residencia las familias inmigrantes. En general, lo suelen hacer en barrios muy concretos de las ciudades, lo que, en la práctica, perjudica en muchos aspectos a los Centros escolares del entorno, produciéndose una 
diáspora de aquellos alumnos autóctonos cuyas familias disfrutan de un nivel económico y social más elevado.

Tabla no 5. Alumnado extranjero matriculado en enseñanzas no universitarias en la Comunidad de Castilla y León por tipo de enseñanza

\begin{tabular}{|l|c|c|c|c|c|c|c|c|c|}
\hline \multicolumn{7}{|c|}{ CIPORSO 2002-2003 } \\
\hline & TOTAL & INFANTIL & PRIMARIA & ESO & BACHILLER & F. P. & E. ESPECIAL & G. SOCIAL & $\begin{array}{c}\text { E.E. RÉGIM. } \\
\text { ESPECIAL }\end{array}$ \\
\hline C. Y LEÓN & 9.693 & 1.533 & 4.094 & 2.547 & 408 & 309 & 16 & 138 & 648 \\
\hline ÁVILA & 535 & 94 & 219 & 164 & 23 & 11 & - & 9 & 15 \\
\hline BURGOS & 1.698 & 321 & 821 & 414 & 38 & 48 & 4 & 17 & 35 \\
\hline LEÓN & 1.555 & 201 & 634 & 504 & 82 & 30 & - & 20 & 84 \\
\hline PALENCIA & 332 & 46 & 162 & 75 & 9 & 15 & 1 & 10 & 14 \\
\hline SALAMANCA & 1.517 & 162 & 427 & 330 & 136 & 85 & 1 & 31 & 365 \\
\hline SEGOVIA & 1.387 & 254 & 634 & 400 & 41 & 28 & 6 & 9 & 15 \\
\hline SORIA & 648 & 139 & 310 & 143 & 13 & 11 & 1 & 12 & 19 \\
\hline VALLADOLID & 1.611 & 269 & 720 & 404 & 57 & 69 & 3 & 10 & 79 \\
\hline ZAMORA & 390 & 47 & 167 & 113 & 9 & 12 & - & 20 & 22 \\
\hline
\end{tabular}

FUENTE: Plan integral de inmigración en Castilla y León 2005-09. Consejería de Presidencia y Administración Territorial. Junta de Castilla y León, 2005, p. 46.

\section{HACIA unA PROPUeSta de aCCIÓn eDUCATIVA: la ATENCIÓN A la DiVERSIDAD CULTURAL}

Nuestro sistema educativo se plantea, no sin urgencia, el logro efectivo de una educación integral, completa, equilibrada, personalizada y adaptada a las características de todos los alumnos sin excepción, como uno de los principios fundamentales de atención a la diversidad cultural. En este sentido, la respuesta educativa pasa, en primer lugar, por comprender la realidad irreversiblemente multicultural de nuestra sociedad (ver Gráfico no 1) y, en segundo lugar, por planificar, diseñar, aplicar y evaluar propuestas y programas de educación intercultural desde una doble perspectiva: a) normalizadora de la acción educativa, y b) colaborativa e integradora de la configuración y desarrollo de un currículo único e interculturalmente enriquecido y compartido.

La educación se ha de entender y se ha de practicar como un elemento sustancial e indispensable para lograr la incorporación e integración de la población inmigrante y para contribuir, de manera especialmente eficaz y activa, a que adquieran y participen, en igualdad de condiciones, de las obligaciones y los derechos del resto de ciudadanos de la Comunidad. 


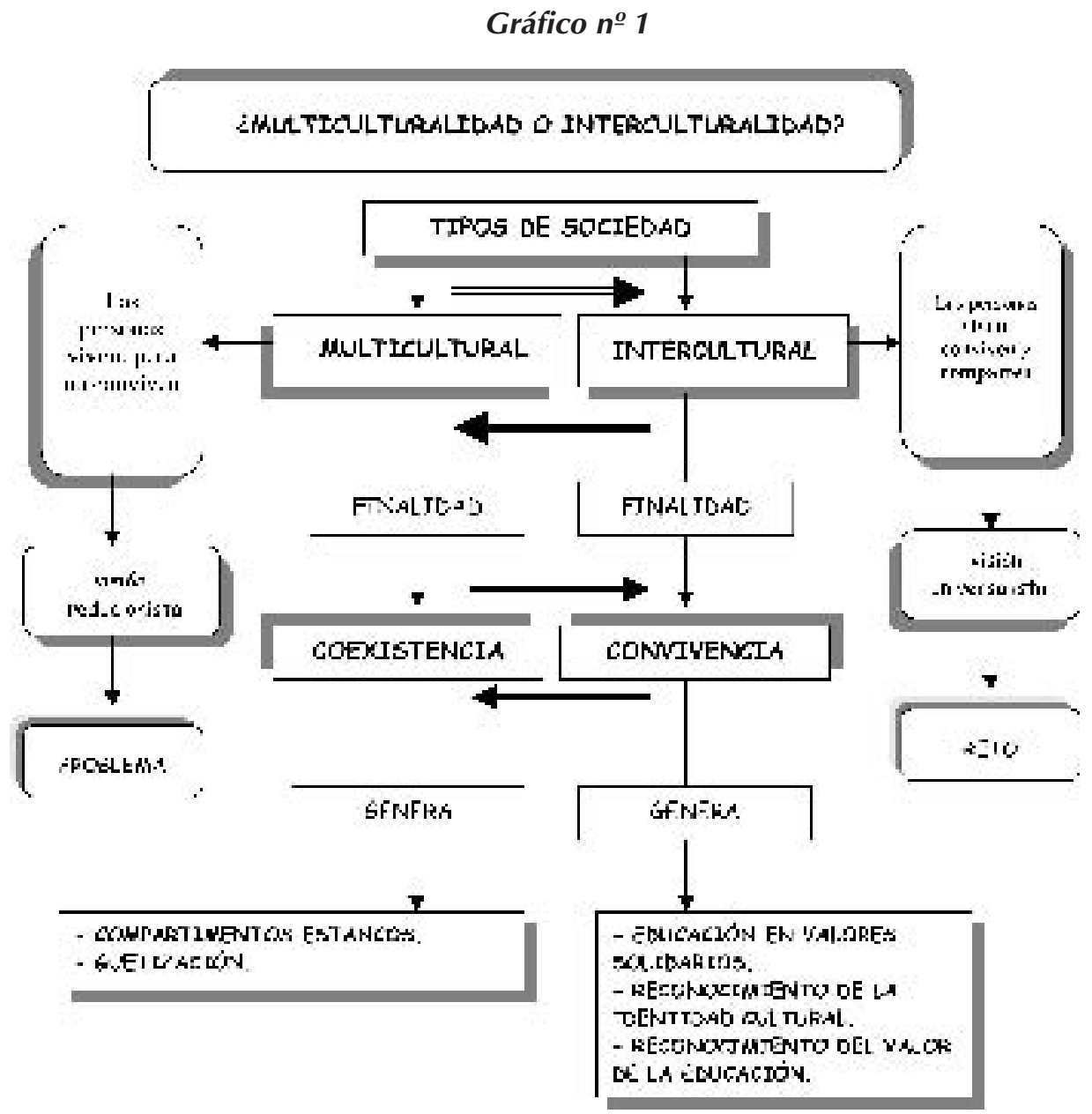

Uno de los desafíos que plantea la inmigración y que con mayor insistencia nos ocupa y preocupa a los educadores es garantizar a este colectivo el derecho a la educación mediante la prestación de un servicio educativo y escolar adecuado, normalizado e integrador, centrado en las necesidades específicas que puede presentar el alumnado extranjero en razón de sus circunstancias socioculturales y dentro de un marco amplio de atención a la diversidad cultural interna y externa.

Por su parte, la Consejería de Educación de la Junta de Castilla y León, especialmente sensibilizada y comprometida con estas cuestiones desde la asunción de las competencias en materia educativa, en enero de 2000, ha venido prestando, con más o menos fortuna hasta el momento, una especial atención a esta incuestionable e irreversible realidad, de manera que, actualmente, podemos decir que está trabajando en esta temática bajo la amplia perspectiva de las necesidades del alumnado, en general, y de las necesidades específicas de compensación educativa, en particular, que presenta esta 
población en desarrollo y con acusada diversidad cultural. Ciertamente esto es así, y así lo certifican las distintas iniciativas y experiencias piloto, -de ellas hablaremos en estas páginas-, que hasta hoy constituyen el mejor referente para asegurar la integración del alumnado de otras culturas en los procesos educativos de nuestros Centros.

Efectivamente, el Plan de Atención al Alumnado Extranjero y de Minorías (aprobado por Orden de la Consejería de Educación con fecha de 29 de diciembre de 2004), en correspondencia con el Marco de Atención Educativa a la Diversidad para Castilla y León, constituye un elemento fundamental en todas estas actuaciones y ha sido objeto de aportaciones desde las diferentes instancias de la comunidad educativa. Su atención se centra en el alumnado inmigrante que presenta necesidades educativas específicas con el fin de garantizar la respuesta educativa más adecuada a sus características personales y a su diversidad cultural. Dentro de esta tipología minoritaria y culturalmente diversa, entre quien se encuentra el grupo o población tradicionalmente perteneciente a minorías étnicas (los gitanos), el alumnado extranjero adquiere particular relieve de cara a intensificar las oportunas medidas educativas y a vertebrar las distintas líneas actuales de trabajo junto con aquellas otras que se prevean para el futuro que, sin duda alguna, comienza hoy.

Por ello, la escuela, como institución inserta en la sociedad y con responsabilidades para con la formación y educación de todos sus ciudadanos sin excepción, no es, no ha de mostrarse impermeable ni puede permanecer ajena a la influencia que sobre ella proyectan los distintos factores sociales y culturales sino que, muy al contrario, se ha de evidenciar especialmente sensible y activa para responder al fenómeno de la multiculturalidad desde una propuesta colaborativa e intercultural de participación integradora.

\section{Medidas educativas de actuación anticipada: el Plan Marco de Atención A LA DiversidAD}

Con la finalidad de hacer posible una atención educativa de calidad para todos los alumnos y, muy especialmente, para aquellos que presentan necesidades educativas específicas asociadas a una, más o menos, acusada diversidad cultural y desventaja socioeconómica, la Consejería de Educación de la Junta de Castilla y León ha promocionado una serie de "medidas educativas de actuación", favorecedoras de experiencias anticipadas de integración inicial y de educación adaptada en una serie de centros de Educación Primaria y Secundaria de su territorio regional, organizadas en torno a unas directrices o ejes fundamentales de actuación conjunta, dentro de un plan marco de atención educativa a la diversidad aprobado mediante Acuerdo de 18 de diciembre de 2003. De estas medidas de actuación, destacamos, especialmente, los indicadores que, a continuación, pasamos a concretar en el siguiente epígrafe.

\subsection{Especificación de las medidas educativas de actuación}

\section{- De identificación y escolarización del alumnado de diversidad cultural}

- Establecimiento de criterios homogéneos de identificación de las necesidades del alumnado con diversidad cultural (alumnos que se encuentran en situación 
de desventaja y presentan necesidades de compensación educativa asociadas a su condición de extranjeros o a una minoría cultural con caracteres fuertemente diferenciados): alumnos en situación de riesgo; alumnos con necesidades educativas específicas; alumnos con necesidades de compensación de aprendizajes básicos; alumnos con necesidades de acceso a la lengua vehicular.

- Valoración regional de las necesidades y de las respuestas educativas.

- Establecimiento de directrices específicas sobre los criterios a seguir en el proceso de escolarización de este alumnado incidiendo en: a) la coordinación entre los distintos niveles educativos, b) la distribución equilibrada del alumnado extranjero y de minorías, c) el tipo de respuesta educativa adaptada a sus necesidades, d) la necesidad de un seguimiento personalizado interno del proceso educativo en el centro.

- Disminución de la ratio en los grupos que escolarizan alumnado con necesidades de compensación educativa: hasta el 20\% del número de alumnos por aula.

- Medidas para lograr la plena escolarización del alumnado extranjero en edad escolar y su permanencia en el sistema educativo: Programa específico para la Prevención y el Control del Absentismo Escolar.

\section{- De integración inicial: elaboración y puesta en funcionamiento de Planes de Acogida}

- Establecimiento de medidas previas, de carácter preparatorio, al momento de acogida, para asegurar el éxito del proceso de integración inicial en el futuro a través de: a) el desarrollo de actividades formativas específicas, b) el acceso a experiencias exitosas de acogida realizadas en otros centros, c) la elaboración de bases para un plan de acogida, d) la organización de intercambios con alumnos de culturas diferentes.

- Diseño de planes de acogida insertos en el marco de las medidas de escolarización general del alumnado asegurando: a) que el alumno recién llegado se sienta aceptado, b) que conozca el contexto y se posibilite la adecuada interacción, c) que se apliquen acciones previstas para asegurar su integración, d) que se exprese la temporalización a seguir y las personas con responsabilidades en el proceso arbitrado, e) que se incardine este plan en el Proyecto Educativo de Centro, f) que se prevean medidas de ajuste de la Planificación General del Centro en cuanto a organización, espacios, horarios y profesorado, g) que se tenga en cuenta la trascendencia de intervenir con rapidez; h) que se preste especial atención a los aspectos lingüísticos, curriculares y actitudinales del alumnado y del contexto, i) que se asegure la coordinación con las familias y con otras instancias implicadas.

- Priorización en la convocatoria de los Planes de Mejora de las propuestas de medidas de acogida por parte de los Centros educativos.

- Actuaciones de carácter informativo dirigidas a las familias del alumnado extranjero por parte de los Centros educativos para: a) facilitar el proceso de adaptación de los nuevos alumnos, b) propiciar el acercamiento, la participación y la integración de las familias en la vida del Centro. 


\section{- De Adaptación Lingüística y Social}

- Generalización de un número suficiente de Aulas de Adaptación Lingüística y Social (Aulas ALISO) en las distintas provincias de Castilla y León, sujetas a una serie de criterios (número de alumnos totales de la provincia, número de alumnos extranjeros, desconocedores del español, ritmo de incremento del alumnado extranjero, distribución provincial de la población extranjera) con la pretensión de: a) proporcionar una rápida adaptación lingüística, b) facilitar el acceso a determinados aspectos culturales y sociales fundamentales, c) permitir la adecuada integración y convivencia con la población del lugar receptor. Cada aula estará dotada de un profesor de apoyo de Educación Compensatoria con perfil adecuado para impartir esta formación lingüística, y estarán ubicadas en los propios Centros educativos.

- Establecimiento de Proyectos de Adaptación Lingüística Inicial en aquellos Centros que no puedan utilizar el recurso del Aula ALISO.

\section{- De atención educativa adaptada para facilitar los procesos de enseñanza/aprendizaje de carácter compensador}

- Medidas de carácter general dirigidas a toda la población escolar del Centro conjuntamente con otras de carácter específico centradas en determinados grupos de alumnos de diversidad cultural.

- Medidas para la educación en valores llevadas a cabo por el profesorado y los tutores (acción tutorial) para el desarrollo de una educación basada en la igualdad de derechos entre todos los alumnos, la convivencia a partir del respeto mutuo y la consideración positiva de la diversidad.

- Proporcionar el contacto directo entre culturas a través de encuentros, convivencias e intercambios entre los alumnos de distintas culturas y países.

- Inclusión en programas de aprendizajes básicos para compensar situaciones de retraso escolar.

- Potenciación de las medidas de apoyo lingüístico.

- Apoyo a programas basados en el reforzamiento de la lengua y cultura del país emisor en colaboración con los diferentes países.

- Asegurar una oferta suficiente de Enseñanzas de Formación Profesional para este alumnado.

- Adopción de medidas de atención educativa individualizada de carácter curricular aplicadas por el profesorado ordinario con el asesoramiento y apoyo de los EOEPs o de los Departamentos de Orientación.

- Potenciación del asesoramiento pedagógico y curricular, de la intervención psicopedagógica y de la actuación socioeducativa.

- Provisión de los recursos humanos y presupuestarios suficientes: un profesor de apoyo específico en cada centro que escolarice más de 20 alumnos con necesidades específicas de compensación debidamente diagnosticadas.

- Apoyo a iniciativas y proyectos de compensación educativa externa. 


\section{- De formación e innovación}

- Creación de un Centro de Recursos de Educación Intercultural (CREI) con carácter regional para prestar apoyo técnico y didáctico a la comunidad educativa, atender al alumnado extranjero y de minorías que presenta necesidades específicas relacionadas con una acusada diversidad cultural y desventaja social o necesidades de compensación educativa. Sus funciones fundamentales son: el desarrollo de actuaciones de educación intercultural; el asesoramiento al profesorado; servir de referencia a los asesores; la preparación de materiales curriculares y extracurriculares; organización de eventos relacionados con estos temas; relación con otras Comunidades Autónomas, etc.

- Impulso a la formación y actualización del profesorado de atención al alumnado de diversidad cultural a través de una serie de instrumentos de apoyo como los Centros de Recursos de Educación Intercultural (CREI), los Centros de Formación e Innovación Educativa (CFIE), los Equipos de Orientación Educativa y Psicopedagógica (EOEP), entre otros.

- Potenciación de experiencias de innovación educativa de atención a la diversidad cultural: experimentación de proyectos novedosos, creativos y enriquecedores.

\section{- De coordinación}

- Integración de los distintos niveles de atención a la diversidad cultural: Centros educativos, Áreas de las Direcciones Provinciales de Educación y Servicios de las Direcciones Generales de la Consejería de Educación.

- Coordinación de las medidas desarrolladas en cada Centro.

- Potenciación de la coordinación interinstitucional mediante la creación de comisiones o foros de trabajo para establecer trabajos y acuerdos conjuntos entre Administraciones o Instituciones.

- Potenciación de la dimensión intercomunitaria de la atención a la diversidad cultural propiciando la coordinación de los especialistas de atención a la diversidad de nuestra Comunidad con expertos y responsables de otras Comunidades del Estado a través de su participación en iniciativas y programas nacionales.

- Potenciación de la dimensión internacional de la atención a la diversidad cultural, mediante la participación en iniciativas y programas, particularmente Europeos, centrados en la Educación Compensatoria e Intercultural.

\section{- De provisión de recursos materiales y personales}

- Dotación preferente de recursos específicos en los Centros con mayores necesidades de atención a la diversidad cultural.

- Potenciación de la dotación de profesores técnicos de Servicios a la Comunidad en los Centros con mayores necesidades de intervención socioeducativa.

- Dotación de recursos para la generalización de las Aulas de Adaptación Lingüística y Social.

- Dotación del Centro de Recursos de Educación Intercultural. 
- Gratuidad del material escolar.

- Consideración especial de las "zonas prioritarias" de atención educativa.

- Entre los responsables de la aplicación de estas medidas, hay que destacar al profesorado generalista, a los profesores de apoyo de Educación Compensatoria, a los profesores técnicos de Formación Profesional de Servicios a la Comunidad en IES, a los Equipos de de Orientación Educativa y Psicopedagógica, a los Departamentos de Orientación, a los asesores de Áreas de Programas Educativos, a los responsables de Áreas de Inspección Educativa, a los asesores de atención a la diversidad de los Centros de Formación e Innovación Educativa (CFIEs).

Junto a estas medidas, afortunadamente enriquecedoras en muchos de sus aspectos de referencia pero, a la vez, incompletas desde mi punto de vista, en relación con la falta o ausencia de elaboración de verdaderos proyectos curriculares de educación intercultural "normalizada", comunes para todo el alumnado de los Centros con minorías culturales en colaboración con toda la comunidad educativa sin excepción, -si es que participamos de un mismo lenguaje pedagógico y didáctico cuando hablamos, en distintos contextos de debate, sobre "educación intercultural"-, la elaboración inicial del Plan objeto de comentario se inicia entre finales de 2003 y principios de 2004, para, seguidamente, proceder a su aplicación de forma progresiva a lo largo de un ciclo de cuatro años, es decir, entre 2004 y 2007 y, finalmente, comenzar a ser evaluado, sucesivamente, a partir del año 2005, según se recoge en el presente cuadro de temporalización (Tabla no 6).

Tabla no 6. Previsiones de temporalización para el desarrollo del Plan de Atención al Alumnado Extranjero y de Minorías en Castilla y León

\begin{tabular}{|l|l|l|l|l|l|}
\hline TEMPORALIZACIÓN & $\mathbf{2 0 0 3}$ & $\mathbf{2 0 0 4}$ & $\mathbf{2 0 0 5}$ & $\mathbf{2 0 0 6}$ & $\mathbf{2 0 0 7}$ \\
\hline ELABORACIÓN DEL & & & & & \\
PLAN DE ATENCIÓN & & & & & \\
AL ALUMNADO \\
EXTRANJERO Y DE \\
MINORÍAS
\end{tabular}

FUENTE: Resolución de 10 de febrero de 2005, de la Dirección General de Formación Profesional e Innovación Educativa, por la que se acuerda la publicación del Plan de Atención al Alumnado Extranjero y de minorías (B.O.C.y L.- №. 49, de 11 de marzo de 2005).

\section{Indicadores de evaluación del Plan de Atención Educativa al Alumnado INMIGRANTE}

Los ejes o indicadores que se proponen para la evaluación de este complejo y urgente Plan de Atención Educativa, arrancan desde una doble perspectiva: por una 
parte, la que tiene como punto de valoración el compromiso de la Administración regional y su implicación en el grado de consecución y logro de los objetivos y proyectos fijados; por otra, la que se refiere al conjunto de las actuaciones y experiencias de innovación puestas en práctica en aquellos contextos escolares de las provincias de la Comunidad especialmente sensibilizadas con el nuevo reto que supone la educación de alumnos inmigrantes. Ambas vías intercomplementarias nos proporcionarán una imagen aproximada del estado de la cuestión y de las perspectivas que se vislumbran a corto plazo. Del resultado de unas y otras dependerán, no solamente las acciones a seguir en adelante sino, también y muy especialmente, la introducción de modificaciones y nuevas propuestas de intervención juntamente con la reformulación de algunas de las ya aplicadas sin el éxito inicialmente previsto. Por ello, para proceder a la valoración general de las actuaciones de atención educativa del alumnado inmigrante en Castilla y León, nos guiaremos por los ejes que se recogen en el Plan anteriormente referido, y que se concretan en:

\section{- Indicadores para la identificación de las necesidades educativas específicas del alumnado extranjero y de minorías}

- Aportación de criterios uniformes y estandarizados para la identificación y valoración del alumnado de diversidad cultural con necesidades educativas específicas.

- Elaboración de instrucciones anuales específicas para este objetivo.

- Protocolo específico para la detección regional de las necesidades educativas específicas.

- Estadística anual del total de alumnos que presentan necesidades educativas específicas asociadas a su acusada diversidad cultural.

- Sistema específico de distribución zonal del alumnado con necesidades de compensación educativa en Castilla y León.

\section{- Indicadores de medidas de integración inicial}

- Pautas para el desarrollo de Planes de acogida en los Centros educativos.

- Generalización de las Aulas de Adaptación Lingüística y Social.

- Creación de 2 Aulas por provincia, como media, independientemente de que las necesidades concretas varíen de unas provincias a otras.

- Determinación de los criterios para reglamentar la distribución de las Aulas de Adaptación Lingüística y Social.

\section{- Indicadores de medidas de atención educativa adaptada}

- Establecimiento de Programas de Aprendizajes Básicos en los Centros que lo necesiten.

- Número de Centros que han puesto en marcha Programas de Aprendizajes Básicos.

- Número de actuaciones de carácter intercultural dirigidas al alumnado ordinario. 
- Número y alcance de actuaciones que logren un contacto directo entre alumnado de distintas culturas y países.

- Número y características de alumnos extranjeros incluidos en acciones de reforzamiento del nivel lingüístico.

- Número de alumnos extranjeros incluidos en programas que supongan el reforzamiento de la lengua y cultura de su país de origen.

- Número de alumnos españoles incluidos en acciones educativas de carácter bilingüe.

- Emisión de instrucciones en las que se ordene el seguimiento de los casos que en algún momento fueron de necesidades educativas específicas asociadas a diversidad cultural, para garantizar un acceso suficiente a programas de preparación personal y profesional, la aplicación de medidas de atención educativa individualizada, de la intervención psicopedagógica, actuación socioeducativa, etc.

\section{- Indicadores de formación e innovación}

- Creación de un Centro de Recursos de Educación Intercultural de carácter regional en el año 2005.

- Intensificación de las acciones formativas sobre atención a la diversidad cultural dirigidas al profesorado de apoyo específico.

- Determinación del número de acciones formativas sobre atención a la diversidad cultural dirigidas al profesorado en general.

- Especificación de materiales de apoyo específicos sobre atención a la diversidad cultural editados y/o difundidos por la Consejería de Educación.

\section{- Indicadores de medidas de coordinación}

- Establecimiento de cauces y alturas de responsabilidad de los distintos niveles de atención a la diversidad cultural.

- Seguimiento de la coordinación interna, realizada en cada Centro educativo, de sus distintas actuaciones referidas a la atención a la diversidad cultural.

- Seguimiento de las acciones de coordinación establecidas con otras Consejerías, Administraciones o Instituciones. Número y características de estas actuaciones.

- Selección, subvención de los proyectos más relevantes sobre la atención educativa complementaria a la diversidad cultural propuestos por entidades y asociaciones sin ánimo de lucro. Un criterio de selección será que no supongan disminución alguna de las acciones educativas desarrolladas en el horario escolar de los alumnos.

- Se llevará una evaluación continua y final de los aspectos progresivamente aplicados a través del nivel de cumplimiento de los indicadores definidos.

\subsection{Resultados de la evaluación de la labor realizada por la Consejería de Educación de la Junta de Castilla y León}

Este proceso, sin duda alguna, debe realizarse teniendo como referencia, en primer lugar, el marco en el que se especifica y se concreta, en términos de acción 
innovadora, el propio concepto de atención educativa a la diversidad cultural, los principios en que se basa, las medidas y acciones previstas acordes con los mismos, la participación de los profesionales en la estructura docente, la implicación y compromiso de la Administración, de las instituciones locales, padres, etc., no como un conjunto de actuaciones desarrolladas de manera específica, sino como un elemento normalizador de referencia inserto en una sociedad plural y multicultural.

Aun cuando el ciclo de experimentación del programa integral de actuación educativa y escolar para la Comunidad de Castilla y León no se haya agotado, sí podemos, no obstante, ofrecer una valoración estimativa de los logros alcanzados hasta el momento, de sus puntos fuertes y débiles y, en consecuencia, del grado de acercamiento entre los objetivos inicialmente previstos, -en formato de "intenciones pedagógicas" -, y lo realmente conseguido en la práctica, de manera que el profesorado tenga la oportunidad de conocer algunos apuntes sobre sus logros y les pueda dar pistas para la participación en Planes de Atención a la Diversidad que, con toda seguridad, tendrán que elaborar todos los Centros para mejorar la atención del alumnado inmigrante.

Un indicador previo a tener en cuenta en este proceso de valoración aproximativa a la labor desarrollada por la Consejería de Educación de la Junta de Castilla y León en el ámbito de la educación intercultural es que el fenómeno migratorio en esta Comunidad es relativamente reciente, ya que sus manifestaciones iniciales no aparecen con cierta claridad y evidencia externa hasta los años 95, es decir, cinco años antes de que la Junta asumiera las competencias educativas que le estaban reservadas.

Entrando ya en materia y situándonos en el año 2003, los datos estadísticos elaborados por la Comunidad de Castilla y León, en un primer trabajo de campo, nos hablan de una población inmigrante de 43.348 personas (pertenecientes a más de 100 países diferentes) dentro de un total de 2.487 .646 habitantes censados, lo que supone el 1,7\% de su índice demográfico. Si esta estimación la referimos al ámbito educativo, la proporción aún sigue siendo moderada a pesar de que el alumnado extranjero, en tan sólo 1 año (curso 2003-04), ha alcanzado la cota del 3\%, a saber: 10.598 inmigrantes escolarizados en el conjunto de los niveles educativos no universitarios, frente a una población estudiantil de 356.932 alumnos. A fecha de hoy (curso 2005-06) las previsiones más rigurosas nos hablan de 15.000 alumnos extranjeros escolarizados, -un 4,2\%-, lo que supone una considerabilísima subida porcentual, en 2 años, respecto del conjunto de la población escolarizada en la Comunidad, y un espectacular aumento del $41 \%$ sobre el global de los residentes extranjeros incluidos en el censo escolar. Datos, estos que, en buena lógica, nos han de hacer reflexionar y pasar a la acción.

Volviendo a los datos ofertados para el curso 2003-04 (a partir de ahora serán los que nos servirán de referencia estimativa), el 46,48\% del alumnado con diversidad cultural (15.889 alumnos extranjeros y de minorías culturales) presenta algún tipo de necesidades educativas específicas, lo que convierte al alumnado extranjero y de minorías culturales en una población escolar de alto riesgo y, consecuentemente, de atención educativa y apoyo especializado.

A estos indicadores, de por sí ya inquietantes, debemos añadir otros que nos permiten acercarnos, con mayor proximidad, a la realidad cualitativa y cuantitativa de 
nuestra Comunidad y de sus Centros y aulas. En este sentido, apuntamos que el $9,14 \%$ de los alumnos no autóctonos se incorporan al sistema educativo sin tener ningún tipo de conocimiento de la lengua propia de este territorio autonómico (el castellano), un $19,36 \%$ posee un nivel bajo, un $42,44 \%$ tiene un nivel medio y el resto, un $29,06 \%$, se sitúa en una banda de conocimiento lingüístico muy superior ya que su nivel de castellano se puede catalogar como de alto.

Actualmente, de todos los países que nos visitan, que son exactamente 106, hay 10 países que aportan el $76 \%$ del alumnado extranjero que se asienta en nuestro territorio Castellano-Leonés. Estos países, por orden de contribución, son los siguientes:

Bulgaria: 1.821 (17,18\%); Ecuador: 1.683 (15,88\%); Colombia: 1.311 (12,37\%); Marruecos: 948 (8,95\%); Portugal: 516 (4,87\%); Rumania: 513 (4,84\%); Argentina: 433 (4,09); República Dominicana: 410 (3,87\%); Brasil: 220 (2,08\%) y Venezuela: $198(1,87 \%)$. Estos alumnos, con sus respectivas familias, eligen distintos lugares de asentamiento, aunque la mayoría, en torno al $61 \%$ de la población extranjera y el $79 \%$ de minorías, se ubica en núcleos de más de 10.000 habitantes y en barrios de las grandes ciudades con carencias estructurales, económicas y sociales, lo que supone, en la mayoría de los casos, una cierta guetización geográfica y escolar.

Dos son las clases de profesores que realizan funciones de compensación educativa e interculturalidad:

- Profesorado de apoyo ordinario: intervienen, dentro de su grupo-clase, con alumnos de compensación educativa mediante refuerzos y apoyos de carácter general. Asimismo, participan, activamente, en las tareas que generan los desdobles de grupo.

- Profesorado de apoyo específico: participan, muy activamente, cuando en un mismo Centro se concentra una población de 25 o más alumnos extranjeros con necesidades de compensación educativa. El número de profesores que participa, específicamente, en actuaciones de compensación educativa e interculturalidad, es de 388, distribuidos de la siguiente manera:

- En Centros públicos: 299. De estos, 222 son Maestros de Educación Infantil y Primaria; 25 son Profesores Técnicos de Servicios a la Comunidad; 33 son Profesores de Centros Rurales de Innovación Educativa, y 19 son Profesores de lengua y cultura portuguesa.

\section{- En Centros privados-concertados: 89.}

Este profesorado atiende, en Centros públicos, al 69,34\% del alumnado extranjero, y en Centros privados-concertados, al 30,66\%, repartidos entre los siguientes niveles de escolarización: Educación Preescolar/Infantil: 1.533 (12,43\%); Educación Primaria: 4.094 (48,99\%); Educación Secundaria: 3.264 (38,58\%). Esta dotación de recursos humanos se ve favorecida y complementada, obviamente, por una partida importante de subvenciones anuales, a cargo de la Consejería de Educación, para el desarrollo de las funciones de estos profesores y para la puesta en funcionamiento de programas complementarios de compensación educativa. Junto a estas acciones hemos de valorar, también, la dotación de recursos técnicos y materiales como son las, hasta ahora, 23 Aulas de Adaptación Lingüística y Social (Aulas ALISO) creadas 
en las distintas provincias; la creación del Centro de Recursos de Educación Intercultural (CREI), como medida de formación didáctica y de apoyo técnico al profesorado y a la innovación de la enseñanza; el establecimiento de proyectos de Adaptación Lingüística Inicial; la elaboración de criterios homogéneos de identificación de las necesidades del alumnado con diversidad cultural; la propuesta de medidas de integración inicial, como los Planes de Acogida; la consideración de acciones de atención educativa adaptada, con especial proyección en los procesos de enseñanza/aprendizaje de carácter compensador y, finalmente, el apoyo especial de las Comisiones de Coordinación Pedagógica, los Tutores, los Profesores de Apoyo Específico y los Orientadores.

Junto a estas acciones, en las que la Junta de Castilla y León ha evidenciado su presencia y apoyo, de manera más persistente desde el año 2000, otras aún están en un proceso más inicial como son las medidas de coordinación, el aumento en la dotación de profesorado autóctono, la aparición en plantilla de profesores extranjeros para la elaboración e implementación de un verdadero currículo intercultural, la sectorización de los centros de integración de alumnos inmigrantes y de sus recursos, entre otras que comentaremos en el apartado de conclusiones.

No obstante, teniendo en cuenta la escasa experiencia de la Junta de Castilla y León en abordar educativamente esta realidad intercultural, hemos de decir, sin lanzar las campanas al vuelo, que su actuación, en general, nos merece una valoración moderadamente positiva aunque con previsiones importantes pendientes de configurar y aplicar.

\subsection{Resultados de la evaluación de la labor realizada por los centros a través de sus experiencias de educación intercultural}

El diseño y desarrollo de proyectos de educación intercultural, con especial predilección hacia la integración educativa y social, y su conocimiento y generalización entre todos y por todos los Centros sin distinción, son elementos motivadores para la potenciación, entre el profesorado, de actitudes positivas hacia la inmigración y de enriquecimiento profesional y docente para aquellos educadores especialmente sensibilizados en compartir expectativas y experiencias comunes.

Las prácticas educativas de carácter extraordinario que se están llevan a cabo para introducir acciones nuevas con el ánimo de afrontar y dar respuesta a la creciente y compleja variedad cultural que aparece en nuestras aulas y centros de la Comunidad de Castilla y León, constituyen un buen referente pedagógico, un modelo convergente de ciudadanía responsable, tolerante y democrática y, a la vez, requieren una revisión para valorar su idoneidad y acercamiento a la finalidad pretendida: atender las necesidades reales de toda la población en edad escolar, de un modo individualizado y, por supuesto, de un modo grupal, desde la creación de espacios, tiempos y contextos culturalmente integradores y favorecedores del valor compensador de la educación.

Ante la imposibilidad temporal de llevar a cabo una evaluación general y puntual de las propuestas e intervenciones implementadas en el conjunto de los Centros públicos y privados-concertados de la Comunidad, hemos optado por tomar una 
muestra suficientemente significativa, por distintas razones pedagógico-didácticas, de manera que el resultado de la valoración que nos merecen sus experiencias de educación intercultural constituyan un fiel reflejo de lo que está sucediendo en este territorio autonómico y, de esta manera, potenciar e incrementar sus puntos fuertes, corregir y mejorar aquellos otros aspectos que se evidencian como más débiles y necesitados de mejora.

Para ello, hemos elaborado un protocolo que, a modo de guión, nos ha servido para volcar los datos almacenados, tras el contacto con estos centros y sus experiencias, a través de los informes a los cuales hemos tenido acceso. Los Centros (Educación Preescolar/Educación Infantil, Educación Primaria, Educación Secundaria y Bachillerato) están ubicados tanto en las capitales como en las poblaciones de ámbito rural más importantes de las distintas provincias castellano-leonesas con una población de alumnos inmigrantes considerable, lo que, sin duda alguna, va a enriquecer, pedagógicamente, el propio valor de sus aportaciones y, evidentemente, la estimación pública del estamento docente, la sensibilidad de la propia Administración y la determinante implicación y colaboración de los padres de todos los alumnos sin excepción: la interculturalidad no es un concepto, reservado al uso y al abuso, exclusivamente teórico de unos pocos, sino, todo lo contrario, es una forma de vida escolar, cultural y social que nos afecta a todos (Tabla $\mathrm{n}^{-}$7).

Tabla no 7. Plantilla de evaluación

\begin{tabular}{|l|l|}
\hline Tipo de Centro & \multicolumn{2}{|l|}{} \\
\hline Educación Preescolar/Educación Infantil & $\mathbf{0}$ \\
\hline Educación Infantil y Primaria & $\mathbf{9}$ \\
\hline Educación Secundaria Obligatoria & $\mathbf{4}$ \\
\hline Bachillerato & $\mathbf{6}$ \\
\hline Entidad & $\mathbf{1 5}$ (2 CRAs) \\
\hline Centro Público & $\mathbf{4}$ \\
\hline Centro Privado-Concertado & $\mathbf{1 8}$ \\
\hline Ponentes & $\mathbf{1 8}$ \\
\hline Maestros & $\mathbf{1}$ \\
\hline Profesores de Secundaria & $\mathbf{1}$ \\
\hline Profesores de Pedagogía Terapéutica & \\
\hline P. Técnicos de Servicios a la Comunidad & $\mathbf{1}$ \\
\hline Profesores de Educación Compensatoria & $\mathbf{1}$ \\
\hline Justificación & $\mathbf{1 7}$ \\
\hline Inclusión en el Proyecto Educativo & $\mathbf{9}$ \\
\hline Protocolo de actuación & \\
\hline Análisis de la realidad contextual & $\mathbf{7}$ \\
\hline Evaluación inicial del alumnado & $\mathbf{1 9}$ \\
\hline
\end{tabular}




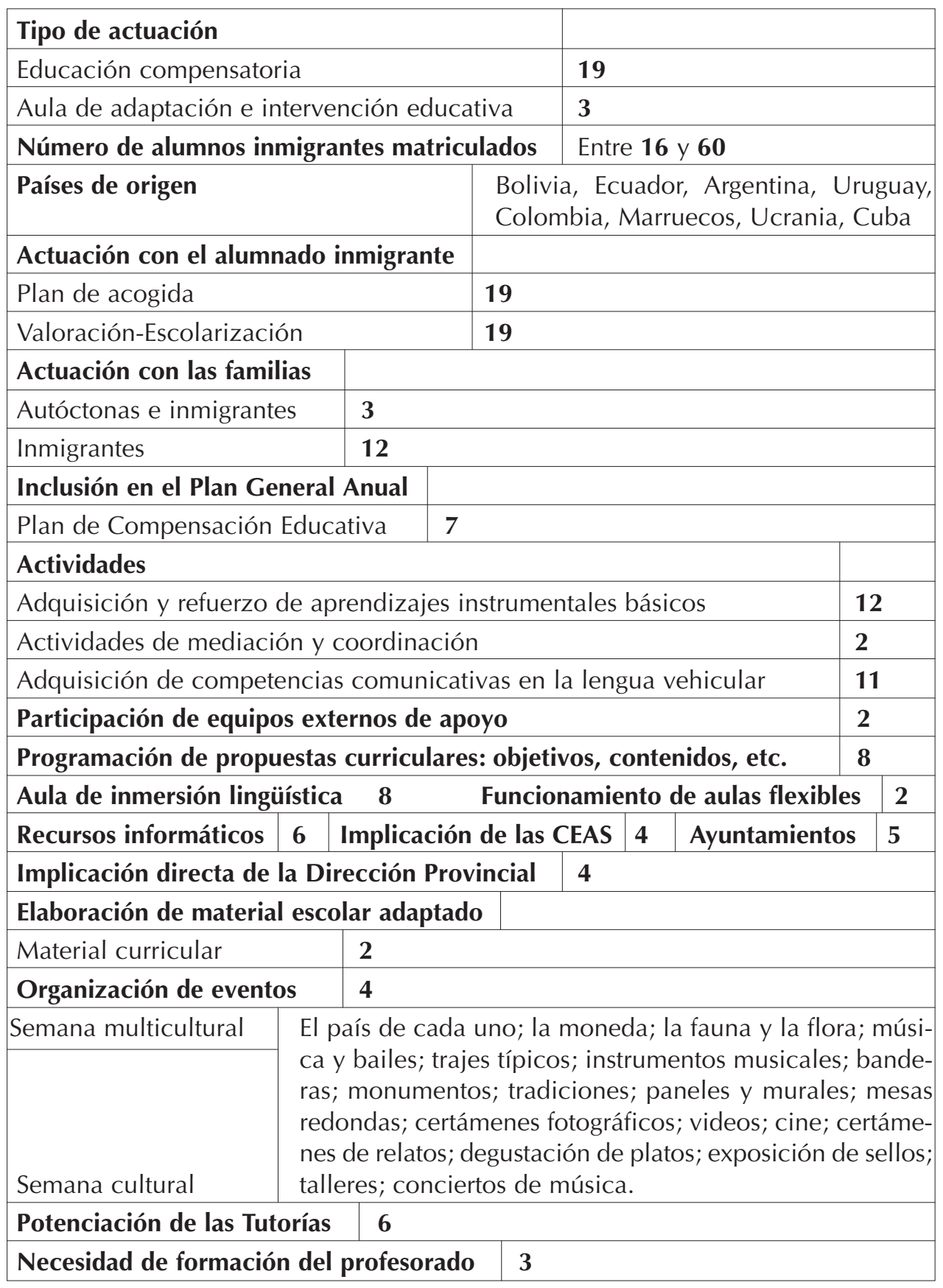

Antes de proceder a comentar y valorar el contenido que aparece reflejado en esta Tabla, nos parece pertinente, aunque el lector haya captado el valor de referencia de sus datos numéricos, indicar que dichos datos expresan el total de Centros, Maestros, 
Proyectos, Protocolos de actuación, Tipos de actuación, Número de inmigrantes, etc., que hemos recogido como consecuencia del estudio llevado a cabo.

Partiendo de un sencillo análisis del contenido que hemos almacenado, a primera vista aparecen una serie de datos ciertamente interesantes y motivadores, como son, en primer lugar, el número de Centros analizados, en total, 19; no son muchos, pero sí que conforman una muestra representativa suficientemente válida en razón del objeto que nos hemos fijado. A partir de esta consideración, podríamos destacar como aportaciones más relevantes, las que, de forma sucinta, aparecen a continuación; el resto se las dejamos a nuestros lectores para su examen y evaluación:

1‥ La participación, en cada Centro, de dos profesores como "Ponentes", además del visto bueno del Director.

2a ${ }^{a}$. La justificación del programa de educación intercultural, en prácticamente todos los Centros, y su contemplación en el "Proyecto Educativo" del 50\% de ellos, lo que contribuye a realzar el carácter que inicialmente adquiere la atención a la población de alumnos extranjeros.

$3^{\text {a }}$. La relevancia que se otorga al "Protocolo de Actuación" en el apartado que se refiere a la "evaluación inicial del alumnado" como garantía de estimación e intervención psicopedagógica y social.

4‥ Respecto del tipo de "Actuaciones" que se llevan a cabo, en todos los Centros y niveles educativos analizados, los "Programas de Educación Compensatoria" colman absolutamente esta modalidad (19 Centros) relegando a un plano muy secundario otras posibilidades, como son las "Aulas de Adaptación e Intervención Educativa" (3 Centros).

5․ "El número de inmigrantes" que frecuentan estos Centros comprometidos con planes experimentales de educación intercultural es, numéricamente, muy importante y, al mismo tiempo, complejo, dada la gran variedad de alumnos de nacionalidades diferentes, aun cuando primen los latinoamericanos sobre el resto (entre un mínimo de 16 y un máximo de 60 alumnos por Centro) y los Colegios de Educación Infantil y Primaria por encima de los Colegios de Educación Secundaria Obligatoria y los IES.

6 a . En cuanto al establecimiento de "Planes de Acogida" y acciones de "Valoración y Escolarización", la satisfacción es máxima puesto que su aplicación se produce y afecta al cien por cien de los Centros implicados.

7ạ. "La actuación con las familias", sobre todo de inmigrantes (12 Centros), se considera una actividad de primera necesidad para mejorar la educación de sus hijos; no ocurre así con las del alumnado autóctono, cuya participación es poco significativa (3 Centros).

8 ${ }^{\text {a }}$. La inclusión del "Plan de Compensación Educativa" en el Plan General Anual de cada Centro, aparece, curiosamente, solamente en el 36\% de los establecimientos analizados, lo que, sin duda alguna, habrá que potenciar para su evaluación y posterior mejora como cualquiera de las acciones ordinarias que, obligatoriamente, se contemplan en dicho documento institucional.

9a. La Adquisición y Refuerzo de "Aprendizajes Instrumentales Básicos" y el logro de "Competencias Comunicativas en la Lengua Vehicular", aparecen 
como dos actividades generalizadas por un alto porcentaje del alumnado con necesidades de compensación educativa y curricular.

10a․ Se hecha en falta una mayor participación de los profesionales de los "Equipos Externos de Apoyo Psicopedagógico", imprescindibles en muchas de las tareas que se suceden en los Centros de integración, sobre todo en aquellas referidas a la evaluación y seguimiento de los alumnos más necesitados y al apoyo técnico que demanda el profesorado implicado.

11a. Es importante, pero no suficiente, que en 8 Centros se trabaje con "Programas Curriculares" a nivel grupal, y no solamente a través de acciones individualizadas y específicas.

12a . En adelante, hay que potenciar la utilización de los recursos informáticos, la implicación directa y sistemática de las Direcciones Provinciales y la colaboración de los Ayuntamientos y CEAS en una acción coordinada y de apoyo complementario de carácter escolar y social.

\section{Conclusiones}

Estos datos conclusivos, aun cuando nos hablan de considerables avances experimentados respecto del conjunto de las medidas tomadas por los Centros, en general, para responder educativamente a la diversidad del alumnado inmigrante, necesitan ser enriquecidos y complementados, no solamente en estos Centros que llevan a cabo este tipo de programas, como es el caso, sino en todos aquellos que escolarizan en sus aulas alumnos de estas características mediante la asunción, con absoluta claridad y comprensión, de una serie de postulados comúnmente consensuados teniendo como referencia el propio concepto de educación intercultural, los principios en que se basa este concepto, las acciones organizativas y curriculares a llevar a cabo y una buena dosis de flexibilidad y autonomía para tomar decisiones y ejecutarlas. En concreto y con carácter específico, proponemos se tengan en cuenta las siguientes consideraciones:

- La educación intercultural, contemplada desde las instituciones escolares, se ha de constituir en el eje motriz que impulse la interrelación entre diversas culturas a través de los programas de integración presentes en todos los Centros. Su correcto desarrollo supone, necesariamente, una atención cuidada de las Administraciones responsables mediante la asignación de fondos suficientes. El apoyo y la ayuda institucional junto con la motivación ilusionada del profesorado y otros profesionales cuya presencia se hace cada vez más necesaria, como, por ejemplo, Educadores Sociales, Trabajadores Sociales, Orientadores, Mediadores Interculturales Escolares, Profesorado oriundo de los países de origen de los alumnos inmigrantes, etc., son requisitos imprescindibles para que dichos programas se generalicen y puedan alcanzar, al menos, gran parte de sus propósitos.

- El proceso de integración e intercambio educativo entre culturas, para que sea completo, se ha de producir, conjuntamente, tanto por parte de la sociedad de acogida, de signo mayoritario, como por el colectivo recién llegado. 
- Pensar en educación intercultural es desarrollar una cultura compartida conciliando intereses individuales y colectivos, valores y actitudes que favorezcan la igualdad de oportunidades y la cohesión social a través de currículos que tengan en cuenta el origen y las culturas del conjunto del alumnado.

- Hay que llegar a la implantación de un modelo (o modelos) de escuela intercultural que desarrolle programas no exclusivamente referidos a los escolares de otras culturas, como viene siendo norma general, sino, fundamentalmente, a todos los alumnos sin mayorías ni minorías.

- Los programas de sensibilización a nivel de padres, profesores, alumnos, etc., han de tener un carácter preferentemente anticipador y favorecedor de encuentros de aprendizaje compartido y de formación participante.

- El currículo escolar debe iniciarse a partir de la cultura de los alumnos, de sus problemáticas, de los contextos en los que están ubicados y desde su realidad.

- Debemos hacer más hincapié en la elaboración de los propios materiales curriculares de aprendizaje para el diseño de un currículo intercultural basado en la heterogeneidad de sus protagonistas y destinatarios, lo más integrado y comprensivo posible, que nos permita y nos facilite la enseñanza cooperativa y la relación interpersonal en pequeños grupos de trabajo colaborativo mediante estrategias flexibles.

- La atención pedagógica y didáctica que demanda la educación intercultural en el escenario de las escuelas e institutos, no se ha de centrar solamente, como viene ocurriendo en la mayoría de las prácticas escolares, en un simple tratamiento y aprendizaje de la lengua de acogida, a ciertos registros-aviso teóricamente explícitos en el Proyecto Educativo de Centro o en acciones momentáneas y puntuales de consideración emocional (Semanas culturales, Días Internacionales de ..., Campañas sobre ..., etc.), sino que se ha de extender y proyectar a todo el alumnado y en todos los momentos de la jornada escolar favoreciendo las aportaciones de las culturas inmigrantes a las materias del currículo que se ha de trabajar y a las actividades complementarias y extraescolares que hay que programar y desarrollar.

- La creación de Aulas Interculturales, como un paso más allá de las actuales Aulas de Inmersión Lingüística, deben facilitar, por un lado, las competencias comunicativas básicas para el alumnado recién Ilegado a los Centros educativos de Castilla y León con un desconocimiento previo del castellano y los conocimientos culturales imprescindibles para su rápida integración social; y, por otro, deben convertirse en aulas donde se impartan acciones dirigidas a que el alumnado y profesorado autóctono conozca los aspectos más importantes de la cultura del alumnado que acoge. Estas aulas no deben desarrollar contenidos curriculares y deberían estar al cargo de personal especializado, docente y no docente.

- La puesta en funcionamiento de Departamentos de Orientación en los Colegios de Educación Infantil y Primaria, junto con el diseño de planes de acción tutorial integrados en el currículo es hoy una urgente y prioritaria necesidad educativa y psicopedagógica imposible de minusvalorar y retardar. 
- La atención a la formación inicial del futuro profesorado desde las Facultades y Centros universitarios de formación, y su posterior formación permanente, se han de concretar, sin más demora, en el primer caso, en una oferta de asignaturas obligatorias y comunes a todas la especialidades del Título de Maestro centradas en la atención a la diversidad cultural y étnica y, en el segundo, en cursos de formación en Pedagogía Intercultural para responder a las necesidades que demandan los profesores que tienen en sus aulas alumnos inmigrantes (Gráfico no 2).

- El intercambio de experiencias educativas, de planes y programas de aplicación entre quienes tienen problemas y proyectos similares, dentro y fuera de la Comunidad Autónoma y con otros países, tiene que ser una tarea normalizada y un modo habitual de enriquecimiento de la acción educativa.

- La preparación y edición de modelos de desarrollo curricular temporal partiendo de la evaluación psicopedagógica y social (nivel de competencias del alumno, estrategias, ritmo de aprendizaje, contexto social y familiar) con proyección sobre los aprendizajes instrumentales básicos, es una labor que han de acometer la Administración educativa, sus técnicos y los profesionales de la educación.

- La atención educativa y escolar en el ámbito en el que nos movemos, precisa de una serie de elementos fundamentales, como son: información sobre experiencias que se están realizando con éxito dentro y fuera de nuestras fronteras nacionales; programas y diseños curriculares innovadores; proyectos y planes experimentales de acción tutorial; programas y material informático; bibliografía y libros de texto representantes de formas de vida y elementos diferentes.

Somos conscientes de que el panorama que hemos dibujado demanda muchas más acciones que las que hemos recogido en estas páginas; no obstante, son éstas las que consideramos, por su urgencia, de mayor necesidad y aplicación más inmediata.

\section{Bibliografía}

ABENOZA GUARDIOLA, R. (2004). Identidad e inmigración: Orientaciones psicopedagógicas. Madrid: MEC/Catarata.

JCyL. (2005). Plan integral de inmigración. Valladolid: Consejería de Presidencia y Administración Territorial. Junta de Castilla y León.

MONTÓN SALES, M. J. (2003). La integración del alumnado inmigrante en el centro escolar. Orientaciones, propuestas y experiencias. Barcelona: GRAO.

QUICIOS GARCÍA, M. P. (2005). Población inmigrante: su integración en la sociedad española (una visión desde la educación). Madrid: Pearson/Prentice Hall.

\section{LEGISLACIÓN}

Resolución de 10 de febrero de 2005, de la Dirección General de Formación Profesional e innovación Educativa, por la que se acuerda la publicación del Plan de Atención al Alumnado Extranjero y de Minorías. Boletín Oficial de Castilla y León, no 49, de 11 de marzo de 2005. 


\section{Gráfico $n^{\underline{0}} 2$}

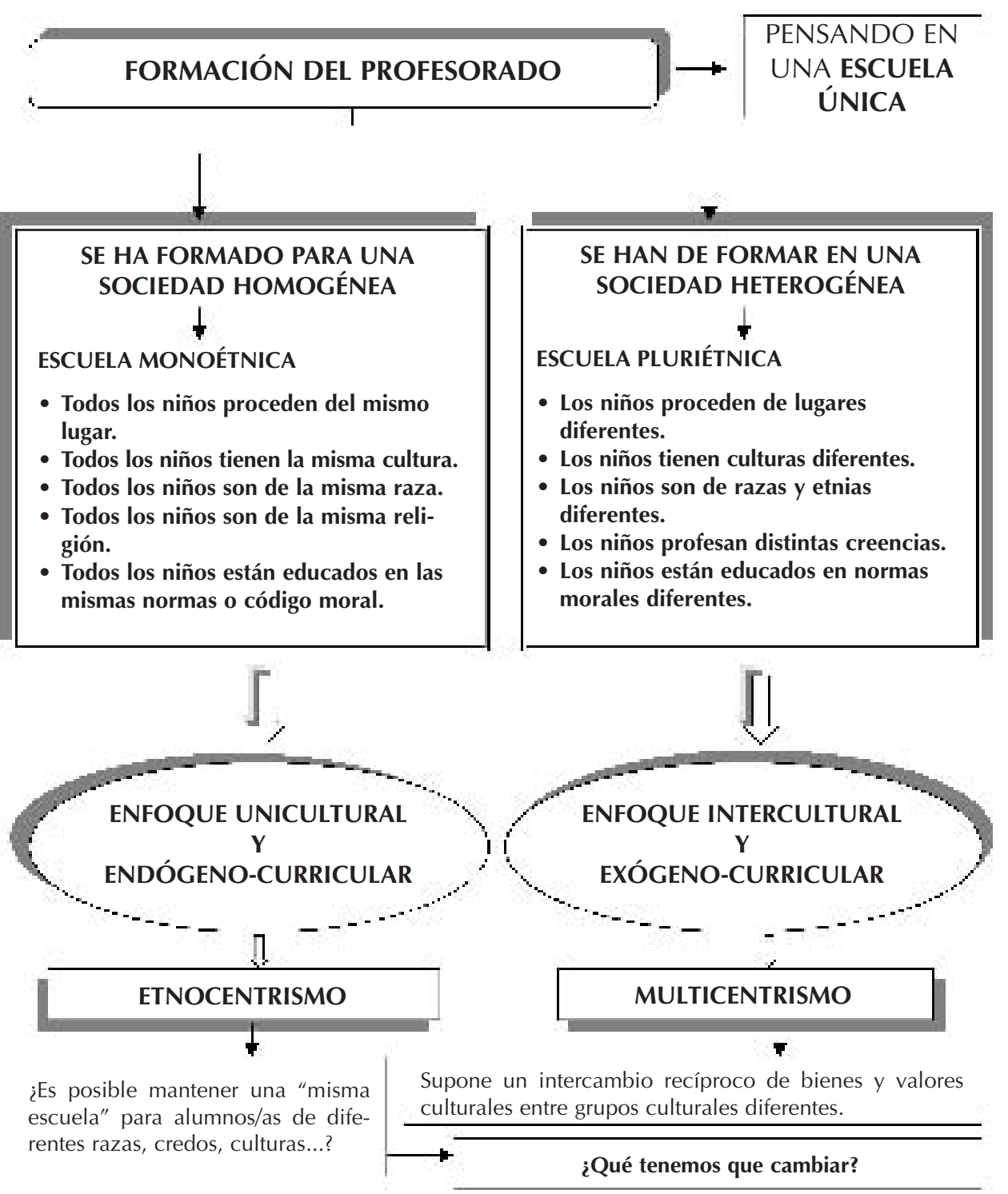

